carotid plaque. Predictive capacity for the presence of plaque by comparing the AUC of the different models was performed through the DeLong method

Results: 106 (38\%), 85 (31\%), 47 (17\%) and 21 (8\%) patients showed respectively SLEDAI null, low, moderate and high, 197 (71\%) had a SLICC >1 and 104 (38\%) a Katz>3. In $36 \%$ (99) of the patients carotid plaques were detected, with an average cIMT of $0.631 \pm 0.108 \mathrm{~mm}$. SLEDAI showed a positive relationship with hypertension; the Katz with hypertension and dyslipidemia; and the SLICC with these and also with age, body mass index and abdominal waist. The relationship of the latter with the CVRF was maintained after subtracting its items related to cardiovascular risk. SLICC was univariatelly related to plaque (OR 1.29 [95\% CI 1.13-1.48], $\mathrm{p}=0.000$ ) and a SLICC $>1$ showed a tendency to be associated with a higher cIMT (beta coefficient 0.03 [95\% $\mathrm{Cl} 0.00-0.06$ ], $\mathrm{p}=0.053$ ). No univariate relationships were found between Katz or SLEDAI with subclinical atheromatosis. The relationship of SLICC with plaque was maintained after adjusting for age, sex and CVRF (OR 1.19 [95\% Cl 1.00-1.42], p=0.047). Similarly, SLICC (even without its vascular damage items) (beta coefficient $0.26[95 \% \mathrm{Cl} 0.12-$ 0.41 ], $p=0.000$ ), but not Katz and SLEDAl, correlated significantly with SCORE. The predictive capacity of SCORE for the presence of plaque was AUC 0.788 (95\% Cl 0.735-0.842). Analogously SLICC showed an AUC 0.659 (95\% Cl 0.594-0.724) for plaque; the AUC of Katz and SLEDAI did not reach statistical significance. The AUC of the SCORE +SLICC versus SCORE did not show statistically significant differences $(p=0.31)$. The statistical significance of the reclassification indexes were net reclasification index $p=0.61$, and integrated discrimination improvement $\mathrm{p}=0.01$.

Conclusions: SLICC is independently related to the presence of plaque. SLE activity, severity and damage indexes are related to CVRF but they have little impact on the predictive capacity of SCORE for the presence of carotid plaque.

Disclosure of Interest: None declared

DOI: 10.1136/annrheumdis-2018-eular.5749

\section{THU0337 NONBACTERIAL THROMBOTIC ENDOCARDITIS (NBTE) IN SLE: PREVALENCE, CLINICAL CHARACTERISTICS AND SEROLOGICAL PROFILE}

D.V. Tanna, M. Bindroo, D. Raval, W. Kazi, S. Bajad, R. Gupta. Rheumatology and clinical immunology, MEDANTA THE MEDICITY, Gurgaon, India

Background: SLE is characterised by excessive production of various autoantibodies and correlation of these antibodies with organ involvement may help to evaluate disease severity and long term prognosis. NBTE is a rare cardiac manifestation of SLE with prevalence rate varying from $6 \%-11 \%$. Many, but no all, studies have shown association of NBTE with anti phospholipid antibodies, but, except this association, data regarding clinical, laboratory and serological characteristics of NBTE is sketchy. We designed this study to evauate profile of patients having NBTE in SLE.

Objectives: 1. To study the prevalenence of NBTE in SLE patients.

2. To study association of NBTE with clinical and laboratory characteristics and serological profile.

Methods: All consecutive SLE inpatients and outpatients attending the department of Rheumatology from September 2015 to December 2017 were enrolled. Patients subjected to 2D Echo were included and their demographic, clinical, laboratory and serological profile were recorded. Serological profile was studied with Blue diver kit which is an immunodot blot assay measuring autoantibodies against 25 ENA. Anti cardiolipin and anti beta 2 glycoprotein antibody were tested by ELISA. Study was approved by an independent ethics committee [ECR/282].

Results: Total number of patients enrolled in study were $\mathbf{3 5 5}$ out of which $\mathbf{2 1 3}$ had undergone 2DEcho. NBTE was found in 33 (15.49\%) patients. Among all autoantibodies studied, we found that the presence of anti-Nucleosome antibody, LAC, ACL and B2GP1 were significantly associated with NBTE $(p<0.05)$. Myocarditis, valvular lesions and Pulmonary Hypertension were more common in NBTE group ( $p$ value: $0.012,<0.0001$ and 0.013 respectively).

We also noticed that there is a statistically significant association between presence of NBTE with APLA syndrome and Thrombotic events ( $p$ value $<0.0001$ and 0.005 respectively).

Tab.1 Significant Serological association of SLE patients with NBTE.

\begin{tabular}{lccc}
\hline Antibodies & SLE With NBTE-33 & SLE without NBTE- & P value \\
& $(15.49 \%)$ & 180 & \\
\hline Anti-nucleosome & $27(81.8)$ & $32(17.7)$ & $<0.0001$ \\
LAC & $16 / 30(53.3)$ & $31 / 149(20.8)$ & 0.0002 \\
ACL (Ig M and IgG) & $9 / 30^{30}$ & $13 / 128(10.1)$ & 0.004 \\
B2GP-1(Ig M and & $6 / 22^{20}$ & $9 / 103(8.73)$ & 0.033
\end{tabular}

IgG)

APLA profile was available in 30 patients of NBTE and 147 patients not having NBTE. Out of this, positivity for APLA antibodies were seen in $17(56.6 \%)$ and 36 (24.4\%) patients respectively [p:0.005]. $82.3 \%$ patients with Anti phospholipid antibodies had APLA syndrome in NBTE group while in NBTE group $48.5 \%$ patients having Anti phospholipid antibodies had APLA syndrome. Thus, presence of NBTE increased the possibility of developing APLA syndrome in patients having positive serology for anti phospholipid antibodies

Tab.2 Significant Clinical and laboratory characteristic of SLE patients with NBTE.

\begin{tabular}{lccc}
\hline Organ & SLE with NBTE- & SLE without NBTE- & p-value \\
involvement & 33 & 180 & \\
\hline Myocarditis & 11 & 27 & 0.012 \\
Valvulopathy & 10 & 4 & $<0.0001$ \\
PAH & 9 & 21 & 0.013 \\
Thrombotic events & 8 & 16 & 0.005 \\
APLA syndrome & 14 & 17 & $<0.0001$ \\
\hline
\end{tabular}

Conclusions: Presence of Anti nucleosome antibody, LAC, Anti cardiolipin and anti beta 2 glycoprotein antibodies may predict presence or future development of NBTE in SLE patients. Presence of NBTE increases probability of developing APLA syndrome in patients with anti phospholipid antibodies. We have found association of NBTE with myocarditis, valvulopathy and PAH and thus propose that such patients with NBTE should be treated early and aggressively.

Disclosure of Interest: None declared

DOI: 10.1136/annrheumdis-2018-eular.5483

\section{THU0338 OUTCOME OF STROKE IN PATIENTS WITH SYSTEMIC LUPUS ERYTHEMATOSUS: A NESTED CASE-CONTROL STUDY}

\section{L.K. Tsoi, C.C. Mok, Y.P. Fu. Medicine, Tuen Mun Hospital, HK, Hong Kong}

Objectives: To evaluate the outcome of stroke in patients with systemic lupus erythematosus (SLE) in comparison with matched non-SLE patients.

Methods: Patients who fulfilled $\geq 4$ ACR criteria for SLE and had a history of stroke were identified from our SLE database. The outcome of stroke in these patients was evaluated retrospectively and compared with a group of randomly selected age/gender-matched non-SLE patients (in a 1:3 ratio) admitted to our stroke unit within the same time period. The type and extent of stroke, atherosclerotic risk factors (hypertension, smoking, diabetes mellitus, dyslipidemia, atrial fibrillation, valvular lesions) and previous stroke were compared between the two groups of patients. The primary outcome of interest was the 90 day functional outcome as assessed by the modified Rankin scale (mRS) (score 0-2: functional independence; score 3-6: functional dependence). Secondary outcomes included all-cause mortality, 30 day stroke mortality, stroke recurrence and stroke complications. Factors independently associated with a poor functional outcome was studied by logistic regression.

Results: A total of 40 SLE patients (age $53.7 \pm 11.5,88 \%$ women) with stroke were identified from our database (stroke prevalence $0.39 / 100$ patient-year). A control group of 120 non-SLE patients (age $52.8 \pm 14.8,87.5 \%$ women) with stroke were randomly selected from our stroke database. All were ethnic Chinese. The prevalence of atherosclerotic risk factors was similar between the two groups except SLE patients had a higher atherogenic index (Log serum [triglyceride/ HDL-cholesterol] (1.51 \pm 0.47 vs $1.32 \pm 0.31 ; p=0.005$.) In SLE patients, the median time to stroke since diagnosis was 24 months. Ischaemic stroke was more common in SLE than non-SLE patients $(90 \%$ vs $63 \% ; p=0.001)$. Among patients with ischaemic stroke, SLE patients had more extensive infarction than controls on CAT scan (multiple infarct $65.7 \%$ vs $18.7 \%$; $p<0.001$ ). The 90 day mRS score was significantly higher in SLE patients than controls $(1.70 \pm 1.97$ vs $0.88 \pm 1.36$; $p=0.004)$. Significantly more SLE patients had functional dependence ( $m R S$ score $3-6)$ at 90 days post-stroke than controls ( 32.5 vs $8.3 \%$; $p<0.001)$. Logistic regression showed that SLE was an independent risk factor for a poor stroke outcome after adjustment for age, sex, history of stroke, various atherosclerotic risk factors and the type of stroke (ischaemic vs haemorrhagic) (OR 12.2 [2.97-49.9]). Subgroup analysis of patients with ischaemic stroke showed that SLE was also independently associated with a poorer functional outcome after adjustment for the same confounding covariates and the extent of stroke (solitary vs multiple infarcts) (OR 12.4 [1.02-150]; $p=0.048$ ). There was no significant difference in the 30 day stroke mortality between SLE and non-SLE patients ( $5 \%$ vs $2.5 \%$; $p=0.43$ ). However, SLE patients had a higher incidence of post-stroke epilepsy than controls (22.5\% vs $3.3 \%$; $p=0.001$ ). Upon a mean follow-up time of $7.5 \pm 5.2$ years, SLE patients had a lower stroke recurrence free survival $(59.5 \%$ vs $85.7 \% ; p<0.001)$ and a higher rate of all-cause mortality (34.6\% vs $15.1 \% ; \mathrm{p}<0.001)$.

Conclusions: Stroke in SLE patients is more likely to be ischaemic in origin and more extensive than matched controls. Short-term functional outcome of stroke is poorer in SLE patients. Over 7.5 years, stroke recurrence, post-stroke epilepsy and all-cause mortality is significantly more frequent in SLE than non-SLE patients.

Disclosure of Interest: None declared

DOI: 10.1136/annrheumdis-2018-eular.6502

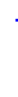

